\title{
Lateral Third Diagonal Branch Artery
}

National Cancer Institute

\section{Source}

National Cancer Institute. Lateral Third Diagonal Branch Artery. NCI Thesaurus. Code C102312.

The lateral branch distal to a bifurcation of the third diagonal artery. 